\title{
Cardiovascular and renal disease burden in type 1 compared to type 2 diabetes: a two-country nationwide observational study
}

Short title: Cardiovascular and renal risk in type 1 and type 2 diabetes

Authors:

Robin Kristófi $\mathrm{MD}^{1}$, Johan Bodegard MD $\mathrm{PhD}^{2}$, Anna Norhammar MD $\mathrm{PhD}^{3}$, Marcus Thuresson $\mathrm{PhD}^{4}$, David Nathanson $\mathrm{MD} \mathrm{PhD}^{5}$, Thomas Nyström MD PhD ${ }^{6}$, Kåre I Birkeland $\mathrm{MD} \mathrm{PhD}^{7}$, Jan W Eriksson $\mathrm{MD} \mathrm{PhD}^{1}$

1. Department of Medical Sciences, Clinical Diabetes and Metabolism, Uppsala University, Uppsala, Sweden; 2. AstraZeneca, Oslo, Norway; 3. Cardiology Unit, Department of Medicine, Solna, Karolinska Institute, Stockholm, Sweden and Capio S:t Göran Hospital, Stockholm, Sweden; 4. Statisticon AB, Uppsala, Sweden; 5. Department of Medicine,

Huddinge, Karolinska Institutet, Stockholm, Sweden; 6. Department of Clinical Science and Education, Karolinska Institutet, Södersjukhuset, Stockholm, Sweden; 7. University of Oslo and Oslo University Hospital, Oslo, Norway

Abstract: 250 words $/ 250$

Manuscript: 3,915 words /4,000

Tables \& Figures: 4/4

\section{Corresponding author}

Dr. Johan Bodegård, MD PhD

AstraZeneca Europe \& Canada

Oslo, Norway

E-mail: johan.bodegard@astrazeneca.com

Telephone no: +4793045811 


\section{Abstract}

Objective: Type 1 (T1D) and type 2 diabetes (T2D) increase risks of cardiovascular and renal disease (CVRD) compared to diabetes-free populations. Direct comparisons between T1D and T2D are scarce. We examined this by pooling full-population cohorts in Sweden and Norway.

Research Design and Methods: 59,331 T1D and 484,241 T2D patients, aged 18-84 years, were followed over a mean period of 2.6 years from December 31, 2013. Patients were identified in nationwide prescribed drug and hospital registries in Norway and Sweden. Prevalence and event rates of myocardial infarction (MI), heart failure (HF), stroke, chronic kidney disease (CKD), all-cause death and cardiovascular death were assessed following age stratification in 5-year intervals. Cox regression analyses were used to estimate risk.

Results: The prevalence of cardiovascular disease was similar in T1D and T2D across age strata, whereas CKD was more common in T1D. Age-adjusted event-rates comparing T1D versus T2D showed that HF risk was increased between the ages 65-79 years, MI between 5579 years and stroke between 40-54 years, 1.3-1.4-fold, 1.3-1.8-fold and 1.4-1.7 fold respectively. CKD risk was 1.4-3.0-fold higher in T1D at all ages. The all-cause death risk was 1.2-1.5-fold higher in T1D above 50 years, with a similar trend for CV death.

Conclusions: Adult T1D compared to T2D patients had an overall greater risk of cardiorenal disease (heart failure and CKD) across ages, of MI and all-cause death at middle-older ages and of stroke at younger ages. The total age-adjusted CVRD burden and risks were greater among T1D patients compared to T2D, highlighting their need for improved prevention strategies. 


\section{Introduction}

In 2019, an estimated 463 million people worldwide were living with diabetes mellitus and the global prevalence is projected to increase by about $50 \%$ by the year 2045.(1) The majority have type 2 diabetes while $5-10 \%$ of diabetes patients suffer from type 1 diabetes. The highest incidence rates of type 1 diabetes can be found in the Scandinavian countries, with disease onset often at a young age and hence a long lifetime with the disease.(2) Globally, cardiovascular (CV) diseases remain the leading cause of death(3), and it has been shown that both type 1 and type 2 diabetes are associated with markedly increased cardiovascular risk compared to populations without diabetes.(4-7) In addition to disturbed glucose metabolism, type 2 diabetes is linked to the metabolic syndrome including obesity, insulin resistance, hypertension and dyslipidemia. These factors are considered to contribute to the accelerated atherosclerotic $\mathrm{CV}$ disease and renal disease (CVRD) development and increased rates that are seen in type 2 diabetes (8), especially represented by cardiorenal disease defined by heart failure and/or CKD.(9) In contrast, these risk factors are not as common in type 1 diabetes patients and their increased CVRD risk has traditionally been explained by the degree and duration of hyperglycemia per se and nephropathy.(10) However, there is a paucity of studies directly comparing the frequency of CVRD between the two major forms of diabetes so it is not known whether the risk differs significantly between these diabetes forms. $(11,12)$

We aimed to directly compare the prevalence and incidence of CVRD in patients with type 1 and type 2 diabetes in large, full-population registries in Sweden and Norway. Furthermore, the presence of chronic kidney disease (CKD), besides being an important microvascular diabetes complication, also in itself is an important risk factor for CV morbidity and mortality. 


\section{Research Designs and Methods}

\section{Data sources}

The present work is part of a large-scale initiative to acquire an understanding of diabetes and its treatment and it is based on the Swedish DAISY and Norwegian DAPHNE studies.(13-16) Both Sweden and Norway have comprehensive, nationwide public health care systems. All citizens have a unique personal identification number (PIN), which is mandatory for administrative purposes, including any contact with the healthcare system as well as prescribed drug dispenses, thus providing comprehensive medical information about the whole population. Individual patient-level data from the Prescribed Drug Registries, the Cause of Death Registries and the National Patient Registries covering all hospitalizations and all out-patient hospital visits, were linked using the PIN, but in a pseudonymized manner. Database management and study protocols are described in the Supplement.

\section{Study population}

All patients with diabetes in Sweden and all patients $>18$ years of age in Norway were identified by using the Prescribed Drug Registries and National Patient Registries. Subjects between the ages of 18 and 84 years at the index date were included in the study population. Type 1 diabetes was defined as having received at least one prescription of short-acting insulin from treatment start or within six months after start of basal insulin treatment and having a hospital diagnosis of type 1 diabetes. In order to validate our definition of type 1 diabetes, we compared our Swedish type 1 diabetes cohort with data from the Swedish National Diabetes Registry (NDR)(17) and from a previous Swedish registry-based study(18) regarding prevalence, incidence, age distribution and age at onset for subjects with type 1 diabetes. We found strong agreements between our cohort and previous data sets in these regards, thus lending support for a valid classification tool (see Supplement Figure S1A-S1D 
for details).

Type 2 diabetes was defined as having any prescribed blood glucose-lowering drug dispensed by a pharmacy, except for those defined as having type 1 diabetes and subjects diagnosed with gestational diabetes or polycystic ovary syndrome according to their respective diagnosis codes in the National Patient Registries. Subjects were followed in the National Patient and Cause of Death Registries until the end of the follow-up period or death.

\section{Baseline and follow-up}

The index date was December 31, 2013 for both countries and the follow-up period was three years for Sweden (until December 31, 2016) and two years for Norway (until December 31, 2015). This amounted to a mean follow-up of 2.6 years and a total of 1.0 million personyears. Baseline comorbidities were searched in the National Patient Registries prior to the index date and baseline drug treatment was searched in the Prescribed Drug Registries one year prior to the index date (presented in detail in Supplement Tables S2-S3).

Age-stratified CV and CKD event rates were calculated during the follow-up period in each country separately and results were then pooled. As the effect of disease duration in type 1 diabetes is known to have a substantial impact on the risk of complications(6), we calculated disease duration for Swedish type 1 diabetes patients and analysed the impact of duration on the various outcomes. Due to registry structure and limited follow-back time, complete data on duration of type 2 diabetes could not be obtained.

\section{Outcomes}

Outcomes were retrieved from in- and outpatient hospital records. In case of inpatient care, diagnoses at the end of the hospital visit were obtained, after completion of clinical and laboratory investigations. Codes from the International Classification of Diseases, Tenth Revision (ICD-10) were used and only first position as main diagnosis was considered (for 
ICD codes of outcomes, see Supplement Table S4). As a sensitivity analysis, all registered diagnosis codes, i.e. also beyond the first position, were searched. The following outcomes were assessed: all-cause death, myocardial infarction (MI), heart failure (HF), stroke, CKD, any CVRD and CV and all-cause death. MI was identified according to the diagnosis code of myocardial infarction. Stroke includes both ischemic and hemorrhagic stroke. HF includes both hypertensive and other heart failure. CKD was defined by a first position diagnosis of diabetic nephropathy, chronic kidney disease or renal failure (hypertensive, acute, chronic or unspecified). CV death was defined as death due to stroke or MI, and any CVRD was defined as any of $\mathrm{HF}, \mathrm{MI}$, stroke, $\mathrm{CV}$ death and CKD. Cardiorenal disease was defined as at least one of $\mathrm{HF}$ or CKD as they often appear together or sequentially. For subgroup analysis of patients with and without previous CVRD at baseline (see below), CKD was defined as described above and CV disease was defined as MI, HF or stroke.

\section{Statistical analyses}

Baseline characteristics were calculated separately for each country and then pooled. Event rates of the outcomes were calculated by dividing number of incident cases by the duration of follow-up in person-years and expressed as incident cases/1000 person-years.

Duration of type 1 diabetes was calculated by retrieving the age at first insulin prescription or first diagnosis registration (whichever came first) available in the National Patient and Prescribed Drug Registries and calculating the time difference to age at the index date. For type 2 diabetes, this was not possible since in most cases there is not a hospital diagnosis, but mainly in primary care where no national registry data are available.

Cox regression models were applied to determine the hazard ratio (HR) and $95 \%$ confidence interval (CI) for each outcome according to type of diabetes. The effect of age was assessed by interaction analysis and was found to have a significant interaction effect on most 
outcomes (not shown). Thus, age-adjusted hazard ratios were calculated within each age category separately. Additionally, we performed further analyses in patients who at baseline were free from previous CVRD and in patients who had a history of previous CVRD. This was done to explore whether previous occurrence of a cardiovascular or renal event would affect the risk in type 1 versus type 2 diabetes for new or recurring events, respectively.

\section{Results}

\section{Baseline data}

A total of 59,331 type 1 diabetes and 484,241 type 2 diabetes patients were included. Data from both countries were pooled as results for the separate country cohorts were comparable. Baseline data are presented in Table 1. At baseline, $87.5 \%$ of type 2 diabetes patients were on any glucose-lowering drug with the remaining patients having previously filled at least one prescription but stopped the treatment before the baseline date. Type 1 diabetes patients were younger than type 2 diabetes patients (mean age 45.8 versus 64.1 years), had a lower overall prevalence of CV disease (13.3\% versus $26.2 \%$ ), a similar proportion of CKD (4.3\% versus $3.3 \%)$ but more frequent microvascular complications (71.6\% versus $27.8 \%)$, mainly driven by increased occurrence of retinopathy (58.6\% versus $13.9 \%$, see Supplement Table S2). A higher use of CV preventive drugs was observed in type 2 diabetes versus type 1 diabetes, possibly due to the higher average age. Following age stratification at baseline, the prevalence of CV disease was similar in both cohorts, with a clear increase from 40 years and above in both type 1 and type 2 diabetes. For MI, HF and stroke, there were no apparent differences in any age groups, as shown in Figure 2. In contrast, the age-dependent increase in prevalence of CKD appeared earlier in type 1 than in type 2 diabetes, and it remained consistently higher in type 1 diabetes across all age groups. The prevalence of all CV diseases combined was slightly higher in type 1 as compared to type 2 diabetes (see Supplement Figure S2). 


\section{Event rates during follow-up}

Results are presented in Figure 3A-F. Event rates were low in age groups below 40 years where comparisons between type 1 and type 2 diabetes could not be performed. For HF, event rates were significantly higher in the type 1 diabetes patients in the ages of 65-79 years (HR values between 1.31-1.39), and for MI there were similar findings in ages 55-79 years (HR values between 1.30-1.79). Rates of stroke, on the other hand, were significantly higher in type 1 diabetes only among younger patients aged 40-54 years (HR values $1.35-1.72$ ). The incidence of CKD was greater for type 1 versus type 2 diabetes at all ages, similar to the baseline prevalence, although inter-group differences generally decreased with increasing age (HR 2.95 for 40-44 years to 1.53 for $80-84$ years). The risk for any CVRD event was significantly higher among type 1 diabetes patients across all age groups (HR 1.20-1.60). CV mortality rates were higher in type 1 than type 2 diabetes at ages 55-64 years and 70-79 years (HR 1.37-1.71, shown in Figure 3), and so were total CVD events at ages 55-79 (HR 1.141.42, shown in Supplement Figure S3). Except for the youngest ages of 40-49 years, all-cause mortality rates were also generally higher in the type 1 diabetes group. As a further sensitivity analysis, we also included all registered diagnosis codes regardless of position, i.e. main as well as additional diagnoses. The comparisons between type 1 and type 2 diabetes were nearly identical to our main analyses (presented in Supplement Figure S4), albeit with the expected higher overall event rates.

\section{Subgroup analyses}

We performed subgroup analyses among subjects who were free of CVRD at baseline and those with previous CVRD (shown in Supplement Figure S5A-H). In the CVRD-free group, the only significant differences between type 1 and type 2 diabetes were increased rates of MI for type 1 diabetes in the age span 70-79 years (HR 1.41-1.70) and CV mortality at 75-79 years (HR 1.78). There were no increased risks of CKD and all-cause mortality among type 1 
diabetes subjects in the CVRD-free cohort as compared to type 2 diabetes. Among subjects with previous CVRD at baseline, results showed similar patterns as the main results in the total cohort (shown in Supplement Figure S6A-H). We also examined the relative impact of disease duration in the Swedish type 1 diabetes cohort and found that it was an important risk factor for CV events and CKD, independent of age per se, presented in Supplement Figure S7. The effect of disease duration was most marked for CKD, but remained substantial for all types of CVRD events. Notably, the effect of duration on all-cause mortality was however much smaller.

\section{Conclusions}

\section{$\underline{\text { Main findings }}$}

In this observational longitudinal full-population study including all patients with type 1 and type 2 diabetes in Sweden and Norway in 2013, we present several important findings on individuals aged 18 to 84 years. First, the age-adjusted incidence of CV diseases were in general higher for type 1 than type 2 diabetes, with a slightly greater stroke risk at younger ages and a greater risk of MI and HF at older ages. Also, CV mortality was greater in type 1 diabetes above 55 years, except the age intervals 65-69 and 80-84 years. Second, CKD was more common in type 1 than type 2 diabetes at all ages. Third, the risk for future CVRD is modified by the presence or absence of pre-existing CVRD, with an almost equal risk in type 1 and type 2 diabetes if CVRD-free at baseline, whereas risks for type 1 diabetes were increased for those with previous CVRD.

This is to our knowledge the first study that has addressed cardiovascular disease in fullpopulation cohorts of both type 1 and type 2 diabetes in modern healthcare systems (from 2013 to 2016). Some studies have reported risks of CV disease(19) and death(11), but these have focused on only one outcome or performed indirect comparisons via populations without 
diabetes.

Previously, Juutilainen et al(11) showed in a long-term small cohort study including 134 type 1 and 834 type 2 diabetes patients that CV mortality was similar in both groups. Subjects in this study were of similar age (45-64 years) and the age of onset was over 30 years. This would suggest that $\mathrm{CV}$ mortality risk is similar in the two diabetes types when taking age into account. Our present study was much larger including nationwide, complete type 1 and type 2 diabetes cohorts which may explain the finding of higher CV mortality in type 1 diabetes over the age of 50 years.

Recently, Fang and colleagues(12) examined data from the U.S. National Health Interview Survey conducted in 2016 and 2017 to compare the burden of complications in young-onset type 1 and type 2 diabetes, defined as being diagnosed before age 40 . They found that there were similar rates of $\mathrm{CV}$ disease between the two groups, which is in agreement with our results, but they also observed similar rates of renal disease, which is different from our findings. This study was purely interview-based, including around 230 subjects with type 1 and 1,200 subjects with type 2 diabetes and statistical power may not have been sufficient to detect true differences between the groups. It is however also possible that young-onset type 2 diabetes represents an aggressive diabetes phenotype with greater cardiorenal risk as previous studies have suggested $(20,21)$, and that this contributed to the increased risk of kidney disease among subjects with type 2 diabetes in the study.

In South Korea, Lee et al(22) followed a population of 20.4 million individuals from 20092016, of whom around 1.9 million had type 2 and 9,400 had type 1 diabetes. In agreement with our results they found a significantly higher risk of MI, HF hospitalization, atrial fibrillation and mortality among the type 1 diabetes subjects as compared to subjects with type 2 diabetes during a mean follow-up of 4.6 years. Furthermore, we show that individuals with type 1 diabetes who already have established CVRD are at a higher risk of further 
CVRD events which suggests that type 1 diabetes patients will benefit from a more intense strategy for both primary and, in particular, secondary prevention of CVRD.

\section{$\underline{\text { Possible explanations for higher CVRD risk in type } 1 \text { diabetes }}$}

Several factors could explain the high risk of CV disease in individuals with type 1 diabetes. Previous studies have established the importance of albuminuria and CKD in increasing the risk of mortality and CV disease in type 1 diabetes. $(23,24)$ We did not have access to laboratory assessments and therefore could not adjust for the degree of renal impairment, but our results indicate that both prevalent and incident CKD is more common in type 1 than in type 2 diabetes and this could contribute to the higher rates of CV disease in type 1 diabetes found in several age groups. We did not have complete data on type 2 diabetes duration, but microvascular complications including diabetic nephropathy, often progressing to manifest $\mathrm{CKD}$, are known to be associated with disease duration(25) and it is likely that the earlier age of onset of type 1 diabetes, and hence longer average duration, would lead to a greater risk of microvascular complications in later life. Hyperglycemia is another risk factor and higher mean $\mathrm{HbA} 1 \mathrm{c}$ levels and long-term glycemic exposure are associated with increased mortality(5) and greater risk of macro- and microvascular complications.(26,27) A recent study based on Swedish National Diabetes Registry data explored the prognostic significance of various risk factors among subjects with type 1 diabetes and found $\mathrm{HbA} 1 \mathrm{c}$ and albuminuria to be the most important predictors for mortality and CV disease.(28) However, other conventional risk factors such as LDL cholesterol and systolic blood pressure were also strongly associated with adverse CV outcomes.

Interestingly, there was an excess risk in the CVRD-free type 1 versus type 2 diabetes cohorts only of MI and CV death in some age strata, but not of other CV events and CKD. The reasons for this are not readily apparent. It is possible that these cohorts were more homogenous with relatively low risks overall. However, it is worth noting that previous 
studies have shown that excess mortality in type 1 diabetes is largely accounted for by nephropathy(24,29), which may explain the finding of a relatively lower risk elevation in type 1 versus type 2 diabetes in the CVRD-free cohorts. In type 1 diabetes, aggressive nephropathy leading to advanced CKD typically has an early onset, and there is seldom development of nephropathy leading to end-stage renal disease (ESRD) after 15 years of type 1

diabetes.(24,30,31) Notably, among type 2 diabetes patients, those with onset at younger ages have a relatively higher risk of serious nephropathy. $(21,30)$ Taken together, these findings are compatible with an increased CKD risk in type 1 diabetes across age strata. However, a similar risk for CKD events in previously CVRD-free subjects with type 1 versus type 2 diabetes is somewhat surprising and warrants detailed further investigations.

\section{$\underline{\text { CVD-preventive treatment }}$}

In our cohort, established drugs to prevent $\mathrm{CV}$ disease such as statins, antihypertensive and antiplatelet drugs were prescribed far less among type 1 diabetes patients compared to type 2 diabetes, albeit largely explained by their overall lower age. This may have contributed to the increased cardiorenal risk in type 1 diabetes. More aggressive intervention with CVD preventive drugs should therefore be considered at an earlier disease stage, and thus at younger ages in type 1 diabetes patients, than what is currently done in routine care. Furthermore, several cardiovascular outcome trials have shown beneficial effects of SGLT2 inhibitors to prevent progression of heart failure and CKD in both medium- and high-risk type 2 diabetes populations.(32-34) SGLT2 inhibitors might be further explored regarding their risk/benefit profile in future interventional outcome studies in type 1 diabetes patients considering their high cardiorenal risk. In type 2 diabetes patients with low-medium CVRD risk, interventional studies should also address initiation of SGLT2 inhibitors at early disease stages. Such studies are currently underway, e.g. in Sweden where the SGLT2 inhibitor dapagliflozin and metformin are compared as first-line therapy in a large randomized 
prospective trial (the SMARTEST trial, ClinicalTrials.gov identifier NCT03982381).

\section{$\underline{\text { Strengths and limitations }}$}

Strengths of the present study include the large population-based cohorts from modern health care covering essentially all type 1 and type 2 diabetes patients in two countries. The representative populations constitute a real-world data set and include patients of a wide age range. To the best of our knowledge, this is the first study in European populations to directly compare cardiovascular and renal complications in type 1 and type 2 diabetes in nationwide full-populations. Importantly, we were also able to validate our definition of type 1 and type 2 diabetes by comparing our type 1 diabetes cohort (concerning prevalence and patient characteristics) with that of the Swedish National Diabetes Registry, showing an essentially complete overlap.

However, our study also has a number of important limitations. First, due to the registry structures and limited follow-back time, it was not possible to accurately determine diabetes duration for all type 2 diabetes patients. Longer duration of type 2 diabetes has been linked with a greater risk of CV disease and death $(36,37)$ and, since we did not have complete information about type 2 diabetes duration, we could not adjust for this. In 2013, i.e. the time of our baseline survey, the median duration in Swedish type 2 diabetes patients was 8 years according to the NDR annual report 2013, also reported in a previous publication.(4) It is clear that diabetes duration overall is longer in our type 1 diabetes cohort, with a median of 14 years (range 0 to $>27$ years) in the Swedish patients. This undoubtedly contributes to the high

CVRD risk in type 1 diabetes, which for several event types overrides that of type 2 diabetes despite its metabolic components. In our cohort of Swedish type 1 diabetes patients for whom duration data were available, we found a higher risk of adverse outcomes in those subjects with a longer age-adjusted type 1 diabetes duration, which is in agreement with previous findings.(38,39) Assuming similar glycemic levels in type 1 and type 2 diabetes and an 
approximate $10 \%$ increase in risk of CVRD for every year of diabetes duration (Supplement Figure S6), the longer average duration of type 1 versus type 2 diabetes at any given age could account for the higher risks we found. Our study did not include a control population. However, previous results comparing type 2 diabetes with the general Swedish population(16) indicated an approximate 1.7-fold increase in the risk for MI and HF, and by extrapolation from our type 2 diabetes cohort we estimate that patients with type 1 diabetes had approximately 2.5 -fold greater risk of MI and HF compared to the background population.

Second, no data were available regarding several important risk factors associated with increased rates of CVRD in diabetes. These include HbA1c, blood lipids, blood pressure and obesity.(24) Furthermore, no information was available regarding socioeconomic and lifestyle risk factors, which are important for outcomes in both type 1 diabetes (40) as well as type 2 diabetes.(41) Such confounding factors should be addressed in future studies.

Third, hospital ICD codes were used and we had no access to primary care data. This may have led to an underestimation of events in type 2 diabetes patients, as the vast majority of them are seen in primary care. However, the main scope of the study was to address risk of major clinical $\mathrm{CV}$ and renal events which almost invariably will lead to in- or outpatient hospital care. For example, early stages of CKD in type 2 diabetes may be underestimated as these patients are typically found in primary care, but the overall occurrence of ESRD was clearly higher among type 1 versus type 2 diabetes patients (Supplement Table S2).

In conclusion, the cardiovascular and renal disease burden amongst type 1 diabetes patients is at least as heavy as in type 2 diabetes patients following age stratification. For several CVRD events like heart failure and MI and clearly for CKD, type 1 diabetes displays the highest risk. There is a remaining need to reinforce and evaluate additional preventive approaches, beyond glycemic control, also in type 1 diabetes patients. 


\section{Article information}

Acknowledgements: We are grateful to Susanna Jerström and Helena Goike at AstraZeneca for logistic support and valuable comments on the manuscript. Urban Olsson, Statisticon AB, is acknowledged for database management. All authors are guarantors of the manuscript. Data from the Norwegian Patient Register, Norwegian Cause of Death Registry and the Norwegian Prescription Database have been used in this publication. The interpretation and reporting of these data are the sole responsibility of the authors, and no endorsement by the Norwegian Patient Register is intended nor should be inferred. Johan Bodegard takes full responsibility for the work as a whole, including the study design, access to data, and the decision to submit and publish the manuscript.

Funding: This work was sponsored and funded by AstraZeneca.

Duality of interest: RK declares no conflicts of interest. TN has received unrestricted grants from AstraZeneca and Novo Nordisk, and is on the national board of Novo Nordisk, SanofiAventis, Eli Lilly and Boehringer Ingelheim. JWE has received research support to his institution from AstraZeneca and for lectures and consulting from Novo Nordisk, Sanofi, AstraZeneca and Merck Sharp \& Dohme. DN has received consultancy fees from Novo Nordisk, Astra Zeneca and Eli Lilly. MT is employed by an independent statistical consultant company, Statisticon AB, Uppsala, Sweden, of which AstraZeneca Nordic-Baltic is a client. AN has honoraria from MSD, Astra Zeneca, Eli Lilly, Boehringer Ingelheim, Novo Nordisk. JB holds a full-time position at AstraZeneca as epidemiologist. KIB has received grants to his institution from AstraZeneca for this study and for lectures and consulting from Novo Nordisk, Sanofi, Lilly, Boehringer Ingelheim, Roche and Merck Sharp \& Dohme.

Prior Presentation: Parts of this study were presented in abstract form at the 79th Scientific Sessions of the American Diabetes Association, San Francisco, CA, 9-11 June 2019. 


\section{References}

1. IIDF. IDF Diabetes Atlas [Internet]. 9th edn. International Diabetes Federation. Brussels, Belgium; 2019. Available from: http://www.idf.org/about-diabetes/factsfigures

2. Katsarou A, Gudbjörnsdottir S, Rawshani A, Dabelea D, Bonifacio E, Anderson BJ, et al. Type 1 diabetes mellitus. Nat Rev Dis Prim. 2017;3.

3. Global Health Estimates 2016: Deaths by Cause, Age, Sex, by Country and by Region, 2000-2016. Geneva, World Health Organization; 2018.

4. Tancredi M, Rosengren A, Svensson AM, Kosiborod M, Pivodic A, Gudbjörnsdottir S, et al. Excess mortality among persons with type 2 diabetes. N Engl J Med. 2015;373(18):1720-32.

5. Lind M, Svensson AM, Kosiborod M, Gudbjörnsdottir S, Pivodic A, Wedel H, et al. Glycemic control and excess mortality in type 1 diabetes. N Engl J Med. 2014;371(21):1972-82.

6. Rawshani A, Sattar N, Franzén S, Rawshani A, Hattersley AT, Svensson AM, et al. Excess mortality and cardiovascular disease in young adults with type 1 diabetes in relation to age at onset: a nationwide, register-based cohort study. Lancet. 2018;392(10146):477-86.

7. Larsson SC, Wallin A, Håkansson N, Stackelberg O, Bäck M, Wolk A. Type 1 and type 2 diabetes mellitus and incidence of seven cardiovascular diseases. Int J Cardiol [Internet]. 2018;262:66-70. Available from: https://doi.org/10.1016/j.ijcard.2018.03.099

8. Low Wang CC, Hess CN, Hiatt WR, Goldfine AB. Clinical update: Cardiovascular disease in diabetes mellitus. Circulation. 2016;133(24):2459-502.

9. Birkeland KI, Bodegard J, Eriksson JW, Norhammar A, Haller H, Linssen GCM, et al. Heart failure and chronic kidney disease manifestation and mortality risk associations in type 2 diabetes: A large multinational cohort study. Diabetes, Obes Metab. 2020;22(9):1607-18.

10. De Ferranti SD, De Boer IH, Fonseca V, Fox CS, Golden SH, Lavie CJ, et al. Type 1 diabetes mellitus and cardiovascular disease: A scientific statement from the American 
Heart Association and American Diabetes Association. Diabetes Care. 2014;37(10):2843-63.

11. Juutilainen A, Lehto S, Rönnemaa T, Pyörälä K, Laakso M. Similarity of the impact of type 1 and type 2 diabetes on cardiovascular mortality in middle-aged subjects. Diabetes Care. 2008;31(4):714-9.

12. Fang M, Echouffo-Tcheugui JB, Selvin E. Burden of complications in U.S. Adults with young-onset type 2 or type 1 diabetes. Diabetes Care. 2020;43(4):E47-9.

13. Lindh A, Persson F, Sobocki P, Bodegard J, Lindarck N. Nordic Longitudinal Data from Electronic Medical Records and Full Population National Registers: Unique Opportunities for New Insights in Benefit of Diabetes Patients. Value Heal. 2015;18(7):A726.

14. Birkeland KI, Bodegard J, Norhammar A, Kuiper JG, Georgiado E, Beekman-Hendriks WL, et al. How representative of a general type 2 diabetes population are patients included in cardiovascular outcome trials with SGLT2 inhibitors? A large European observational study. Diabetes, Obes Metab. 2019;21(4):968-74.

15. Birkeland KI, Jørgensen ME, Carstensen B, Persson F, Gulseth HL, Thuresson M, et al. Cardiovascular mortality and morbidity in patients with type 2 diabetes following initiation of sodium-glucose co-transporter-2 inhibitors versus other glucose-lowering drugs (CVD-REAL Nordic): a multinational observational analysis. Lancet Diabetes Endocrinol. 2017;5(9):709-17.

16. Norhammar A, Bodegård J, Nyström T, Thuresson M, Eriksson JW, Nathanson D. Incidence, prevalence and mortality of type 2 diabetes requiring glucose-lowering treatment, and associated risks of cardiovascular complications: a nationwide study in Sweden, 2006-2013. Diabetologia [Internet]. 2016;59(8):1692-701. Available from: http://dx.doi.org/10.1007/s00125-016-3971-y

17. Swedish National Diabetes Register Annual Report 2017. Available on: https://www.ndr.nu/pdfs/Arsrapport_NDR_2017.pdf. Accessed: 2020-10-25.

18. Rawshani A, Landin-Olsson M, Svensson AM, Nyström L, Arnqvist HJ, Bolinder J, et al. The incidence of diabetes among 0-34 year olds in Sweden: New data and better methods. Diabetologia. 2014;57(7):1375-81. 
19. McAllister DA, Read SH, Kerssens J, Livingstone S, McGurnaghan S, Jhund P, et al. Incidence of hospitalization for heart failure and case-fatality among 3.25 million people with and without diabetes mellitus. Circulation. 2018;138(24):2774-86.

20. Svensson M, Sundkvist G, Arnqvist H, Björk E, Blohmé G, Bolinder J, et al. Signs of Nephropathy May Occur Early in Young Adults With Diabetes Despite Modern Diabetes Management: Results from the nationwide population-based Diabetes Incidence Study in Sweden (DISS). Diabetes Care. 2003;26(10):2903-9.

21. Constantino MI, Molyneaux L, Limacher-Gisler F, Al-Saeed A, Luo C, Wu T, et al. Long-term complications and mortality in young-onset diabetes: Type 2 diabetes is more hazardous and lethal than type 1 diabetes. Diabetes Care. 2013;36(12):3863-9.

22. Lee Y Bin, Han K, Kim B, Lee SE, Jun JE, Ahn J, et al. Risk of early mortality and cardiovascular disease in type 1 diabetes: A comparison with type 2 diabetes, a nationwide study. Cardiovasc Diabetol [Internet]. 2019;18(1):1-17. Available from: https://doi.org/10.1186/s12933-019-0953-7

23. Miller RG, Costacou T, Orchard TJ. Risk factor modeling for cardiovascular disease in type 1 diabetes in the pittsburgh epidemiology of diabetes complications (EDC) study: A comparison with the diabetes control and complications trial/epidemiology of diabetes interventions and complication. Diabetes. 2019;68(2):409-19.

24. Groop PH, Thomas MC, Moran JL, Wadèn J, Thorn LM, Mäkinen VP, et al. The presence and severity of chronic kidney disease predicts all-cause mortality in type 1 diabetes. Diabetes. 2009;58(7):1651-8.

25. Drummond KN, Kramer MS, Suissa S, Lévy-Marchal C, Dell'Aniello S, Sinaiko A, et al. Effects of duration and age at onset of type 1 diabetes on preclinical manifestations of nephropathy. Diabetes. 2003;52(7):1818-24.

26. Lund SS, Rossing P, Vaag AA. Follow-up of intensive glucose control in type 2 diabetes. N Engl J Med. 2009;360(4):416-8.

27. Nathan DM. The diabetes control and complications trial/epidemiology of diabetes interventions and complications study at 30 years: Overview. Diabetes Care. 2014;37(1):9-16.

28. Rawshani A, Rawshani A, Sattar N, Franzén S, Mcguire DK, Eliasson B, et al. Relative 
Prognostic Importance and Optimal Levels of Risk Factors for Mortality and Cardiovascular Outcomes in Type 1 Diabetes Mellitus. Circulation. 2019;139(16):1900-12.

29. Orchard TJ, Secrest AM, Miller RG, Costacou T. In the absence of renal disease, 20 year mortality risk in type 1 diabetes is comparable to that of the general population: A report from the Pittsburgh Epidemiology of Diabetes Complications Study. Diabetologia. 2010;53(11):2312-9.

30. Svensson MK, Tyrberg M, Nyström L et al. The risk for diabetic nephropathy is low in young adults in a 17-year follow-up from the Diabetes Incidence Study in Sweden (DISS). Older age and higher BMI at diabetes onset can be important risk factors. Diabetes Metab Res Rev. 2015;31:138-146.

31. Helve J, Sund R, Arffman M, Harjutsalo V, Groop PH, Gronhagen-Riska C, et al. Incidence of end-stage renal disease in patients with type 1 diabetes. Diabetes Care. 2018;41(3):434-9.

32. Zinman B, Wanner C, Lachin JM, Fitchett D, Bluhmki E, Hantel S, et al. Empagliflozin, cardiovascular outcomes, and mortality in type 2 diabetes. N Engl J Med. 2015;373(22):2117-28.

33. Wiviott SD, Raz I, Bonaca MP, Mosenzon O, Kato ET, Cahn A, et al. Dapagliflozin and cardiovascular outcomes in type 2 diabetes. N Engl J Med. 2019;380(4):347-57.

34. Furtado RHM, Bonaca MP, Raz I, Zelniker TA, Mosenzon O, Cahn A, et al. Dapagliflozin and Cardiovascular Outcomes in Patients With Type 2 Diabetes Mellitus and Previous Myocardial Infarction: Subanalysis From the DECLARE-TIMI 58 Trial. Circulation. 2019;139(22):2516-27.

35. Birkeland KI, Bodegard J, Banerjee A, Kim DJ, Norhammar A, Eriksson JW, et al. Lower cardiorenal risk with sodium-glucose cotransporter-2 inhibitors versus dipeptidyl peptidase- 4 inhibitors in patients with type 2 diabetes without cardiovascular and renal diseases: A large multinational observational study. Diabetes, Obes Metab. 2020;

36. Zoungas S, Woodward M, Li Q, Cooper ME, Hamet P, Harrap S, et al. Impact of age, age at diagnosis and duration of diabetes on the risk of macrovascular and microvascular complications and death in type 2 diabetes. Diabetologia. 
2014;57(12):2465-74.

37. Huo L, Magliano DJ, Rancière F, Harding JL, Nanayakkara N, Shaw JE, et al. Impact of age at diagnosis and duration of type 2 diabetes on mortality in Australia 19972011. Diabetologia. 2018;61(5):1055-63.

38. Pambianco G, Costacou T, Ellis D, Becker DJ, Klein R, Orchard TJ. The 30-year natural history of type 1 diabetes complications: The Pittsburgh epidemiology of diabetes complications study experience. Diabetes. 2006;55(5):1463-9.

39. Soedamah-Muthu SS, Chaturvedi N, Witte DR, Stevens LK, Porta M, Fuller JH. Relationship between risk factors and mortality in type 1 diabetic patients in europe: The eurodiab prospective complications study (PCS). Diabetes Care. 2008;31(7):1360_ 6.

40. Secrest AM, Costacou T, Gutelius B, Miller RG, Songer TJ, Orchard TJ. Associations Between Socioeconomic Status and Major Complications in Type 1 Diabetes: The Pittsburgh Epidemiology of Diabetes Complication (EDC) Study. Ann Epidemiol. 2011;21(5):374-81.

41. Tatulashvili S, Fagherazzi G, Dow C, Cohen R, Fosse S, Bihan H. Socioeconomic inequalities and type 2 diabetes complications: A systematic review. Diabetes Metab [Internet]. 2020;46(2):89-99. Available from: https://doi.org/10.1016/j.diabet.2019.11.001 


\section{Tables}

Table 1. Baseline characteristics of type 1 and type 2 diabetes patients in Sweden and Norway in 2013 


\begin{tabular}{|c|c|c|}
\hline & $\begin{array}{l}\text { Type } 1 \text { diabetes } \\
(\mathrm{n}=59,331)\end{array}$ & Type 2 diabetes $(n=484,241)$ \\
\hline Age in years, mean & $45.8(16.5)$ & $64.1(12.4)$ \\
\hline$<50$ years & $34,405(58.0 \%)$ & $64,365(13.3 \%)$ \\
\hline $50-75$ years & $22,978(38.7 \%)$ & $315,495(65.2 \%)$ \\
\hline$>75$ years & $1,948(3.3 \%)$ & $104,381(21.6 \%)$ \\
\hline Sex, females & $24,911(42.0 \%)$ & $208,361(43.0 \%)$ \\
\hline Cardiovascular disease & $7,913(13.3 \%)$ & $126,769(26.2 \%)$ \\
\hline Myocardial infarction & $2,476(4.2 \%)$ & $41,784(8.6 \%)$ \\
\hline Heart failure & $1,867(3.1 \%)$ & $36,520(7.5 \%)$ \\
\hline Atrial fibrillation & $1,706(2.9 \%)$ & $46,626(9.6 \%)$ \\
\hline Stroke & $2,570(4.3 \%)$ & $45,074(9.3 \%)$ \\
\hline Peripheral artery disease & $2,987(5.0 \%)$ & $28,415(5.9 \%)$ \\
\hline Chronic kidney disease & $2,530(4.3 \%)$ & $15,985(3.3 \%)$ \\
\hline Microvascular complications & $42,452(71.6 \%)$ & $134,530(27.8 \%)$ \\
\hline Severe hypoglycemia & $1,762(3.0 \%)$ & $5,349(1.1 \%)$ \\
\hline Cancer* & $2,237(3.8 \%)$ & $45,505(9.4 \%)$ \\
\hline CVD risk treatment & $31,961(53.9 \%)$ & $397,795(82.1 \%)$ \\
\hline Low dose aspirin & $11,530(19.4 \%)$ & $172,529(35.6 \%)$ \\
\hline Statins & $23,782(40.1 \%)$ & $281,159(58.1 \%)$ \\
\hline Antihypertensives & $24,604(41.5 \%)$ & $348,975(72.1 \%)$ \\
\hline Diabetes medication & $59,331(100.0 \%)$ & $423,747(87.5 \%)$ \\
\hline Metformin & $2,043(3.4 \%)$ & $344,651(71.2 \%)$ \\
\hline Sulfonylurea & $57(0.1 \%)$ & $89,733(18.5 \%)$ \\
\hline DPP-4i & $224(0.4 \%)$ & $34,722(7.2 \%)$ \\
\hline SGLT-2i & $30(0.1 \%)$ & $2,021(0.4 \%)$ \\
\hline GLP-1RA & $312(0.5 \%)$ & $17,677(3.7 \%)$ \\
\hline Insulin & $59,331(100.0 \%)$ & $134,350(27.7 \%)$ \\
\hline
\end{tabular}


Data are $\mathrm{n}(\%)$ or mean (SD), unless otherwise stated. DPP-4i: dipeptidyl-peptidase-4 inhibitors SGLT-2i: sodium-glucose-cotransporter-2-inhibitors. GLP-1RA: glucagon-like peptide-1 receptor agonists.

*Cancer diagnosis within 5 years prior to index date.

Microvascular complications include retinopathy, peripheral angiopathy, diabetic foot, neuropathy and nephropathy.

\section{Figure legends}

Figure 1. Flow chart for patients included in the study at the index date 31st of December 2013

Figure 2. Age-stratified baseline prevalence of chronic kidney disease (CKD), heart failure (HF), cardiorenal disease (HF or CKD), stroke, myocardial infarction (MI) and atherosclerotic cardiovascular disease (ASCVD; MI or stroke)

Figure 3. Age-stratified incidence of any cardiovascular or renal diseases (CVRD; including MI, stroke, HF, CKD or CV death), cardiorenal disease (HF or CKD), heart failure (HF), chronic kidney disease (CKD), stroke, myocardial infarction (MI) all-cause death, and cardiovascular death. 